\title{
Research on lightning stroke model and characteristics of intelligent transformer
}

\author{
$\mathrm{Mu} \mathrm{LI}^{1}$, Tao $\mathrm{LI}^{1}$, Jia Jie WANG ${ }^{1}$ and Ling Hong TAO ${ }^{1}$ \\ ${ }^{1}$ Wuhan NARI Limited Liability Company, State Grid Electric Power Research Institute, Wuhan 430074, China
}

\begin{abstract}
In order to improve the reliability of power supply, it is necessary to study the intelligent transformer of the key equipment in the smart substation. Firstly, the transformer model based on the magnetic coupling mechanism is given according to the magnetic coupling characteristics of the transformer. Then, considering the static coupling characteristics of the transformer under high frequency conditions, an improved transformer model based on wide frequency mechanism is obtained. Finally, the effectiveness of the proposed model is verified by comparing the simulation data with the actual measured data for an actual object.
\end{abstract}

\section{Introduction}

With the development and application of sensor technology and information technology, the transformation from traditional substation to smart substation has gradually become a reality. Transformer is one of the key equipment in smart substation.

To ensure the safe and reliable operation of the transformer, it is necessary to comprehensively and accurately grasp the characteristics of the over voltage surge in lightning wave shows, according to the characteristics of lightning and risk to develop the corresponding protective measures and strategies. Now we focus on analyzing the lightning stroke model of transformer and its lightning characteristics ${ }^{[1-3]}$.

\section{Model of intelligent transformer}

Compared with the transformer in conventional substation, there is no special design for the transformer body structure in the smart substation. It only collects the measured values of various sensors directly into the merging unit near the transformer, and then transforms it to the optical signal and then uploads it to second-order system equipment of the main control room through the optical cable.

In order to analyze the influence of lightning on the intelligent transformer more accurately, we extend the simplified transformer model, build a more refined transformer model, and build a lightning stroke model of intelligent transformer combined with sensors ${ }^{[4-5]}$.

In order to obtain the wide frequency characteristic of transformer, it is necessary not only to analyze the magnetic coupling between transformer windings in low frequency, but also to consider the static coupling between transformer conductors under high frequency. In fact, with the increase of frequency, the distributed capacitance parameters which are closely related to the structure size of the transformer will affect the operating characteristics of the transformer, and interact with the power electronic devices on both sides, therefore, the distributed capacitance of the transformer can not be ignored. Therefore, the traditional low frequency transformer model is no longer applicable because it can not take into account the capacitance effect in the transformer. Figure. 1 is the circuit diagram of a single phase double winding transformer.

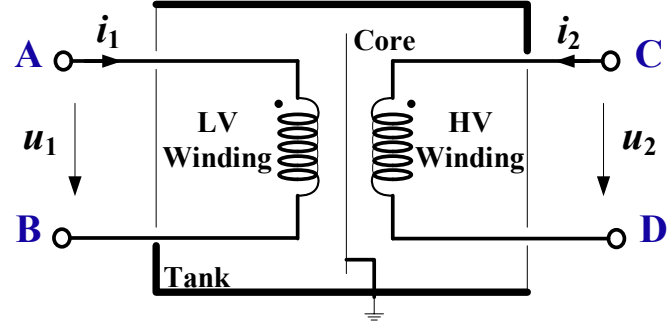

Figure 1. Circuit diagram of transformer

With the development of flexible DC converter system to high voltage and large capacity, the capacity and voltage level of transformer will be improved, the turn number of transformer winding becomes larger, and the oil immersed type is needed to meet the insulation demand in the high-power field. In addition, in order to improve the power density and reduce the loss of the transformer, some magnetic materials with low loss density and high permeability, such as ultra-thin silicon steel sheet and nanocrystalline alloy, are also used as transformer cores, and these core materials are also conductors. Therefore, it is necessary to analyze the capacitance effect between transformer winding, magnetic core and fuel tank.

The electrical characteristics of transformer at low frequency mainly depend on the magnetic coupling 
between transformer windings, but in the high frequency section, the electrostatic coupling between transformer winding, magnetic core and oil tank can not be ignored. Therefore, in order to establish the broadband mechanism model of transformer, it is necessary to deeply analyze the magnetic coupling and electrostatic coupling characteristics of the transformer.

\subsection{Model based on magnetic coupling mechanism}

Because we focus on the external characteristics of the transformer, it is considered that the magnetic core of the transformer operates in the coupled linear region. The coupling inductance model of the transformer is shown in figure 2(a).

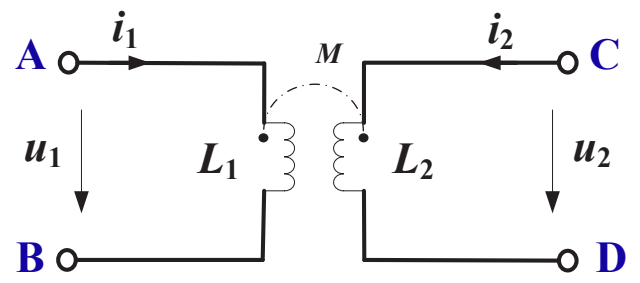

(a) Model with coupled inductors

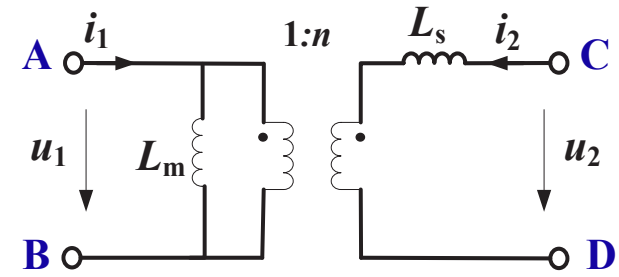

(b) Equivalent model with ideal transformer

Figure 2. Transformer model based on Lossless magnetic mechanism

The inductance matrix of transformer winding can be fully expressed by self inductance and mutual inductance, and the leakage inductance parameter from C-D side can be expressed as:

$$
L_{s}=L_{2}-M^{2} / L_{1}=\left(1-k^{2}\right) L_{2}
$$

Here, $\mathrm{k}$ is the coupling coefficient of transformer windings. Due to the existence of the magnetic core of the transformer, the $\mathrm{k}$ is usually close to 1 .

Because the leakage inductance is represented as the difference between the two large numbers, the leakage inductance obtained by equation 1 may not be accurate. Considering the leakage inductance will affect the impedance characteristics of the transformer in high frequency, we adopt an equivalent model with ideal transformer, as shown in Figure 2 (b). $\mathrm{Lm}$ is the excitation inductance from the A-B side, and the $\mathrm{n}$ is the ideal transformer ratio:

$$
\begin{aligned}
& L_{m}=L_{1} \\
& \mathrm{n}=\mathrm{k} \sqrt{\frac{L_{2}}{L_{1}}}
\end{aligned}
$$

The equivalent relation of the two transformer models in Figure 2 can be proved by the Kirchhoff current law of the primary side of transformer and the Kirchhoff voltage law of the secondaly side of the transformer. Using the model with ideal transformer as shown in Figure 2 (b), the leakage inductance can be taken as an independent variable of the model, and can be directly extracted by magnetic field analysis method. At the same time, the mutual inductance is eliminated so that the circuit analysis of the transformer can be easily carried out.

For an actual transformer, winding and core losses need to be considered to improve the accuracy of the model. On the basis of the model shown in Figure 2 (b), two winding resistances Rs1 and Rs2 are added, and the magnetic core loss equivalent resistance $\mathrm{Rm}$ is parallel to the excitation branch, and finally the final magnetic mechanism model is shown in Figure 3. The model is a low frequency model of traditional transformer, and has been widely used in the field of power system and motor. However, the model is not suitable for overvoltage analysis of transformer because it does not consider the capacitance effect between winding, magnetic core and tank.

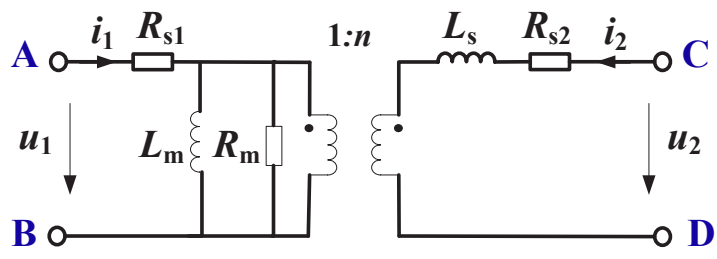

Figure 3. Transformer model based on magnetic mechanism

\subsection{Model based on electrostatic coupling mechanism}

The distributed capacitance is closely related to the electrostatic coupling between the conductors in the transformer. The conductors in transformer include low voltage winding conductor, high voltage winding conductor, magnetic core and oil tank. Due to the voltage drop between the conductors of the transformer, there is a coupling capacitance between these conductors. Considering the low-voltage winding voltage, high voltage winding voltage and winding voltage, the static characteristics of the winding can be described by six capacitors, as shown in Figure 4. However, this model does not consider the electrostatic coupling between winding, magnetic core and fuel tank.

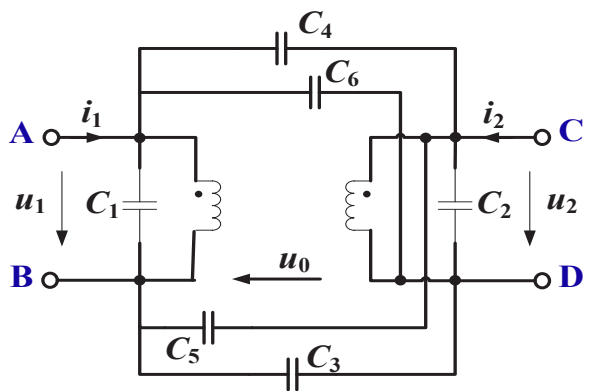

Figure 4. Model with six capacitor

So we propose a transformer model considering the electrostatic coupling mechanism of capacitance, as shown in figure 5 . The core is connected to the tank and connected to the ground.

In Figure 5, in addition to six capacitors representing the electrostatic characteristics of the transformer 
windings, new four capacitors $\mathrm{C} 7, \mathrm{C} 8, \mathrm{C} 9$ and $\mathrm{C} 10$, are also introduced between the winding terminals and the core and the fuel tank to reflect the capacitance effect between the winding, the core and the tank. By adding these capacitors, the electrostatic coupling characteristics of the transformer are fully considered.

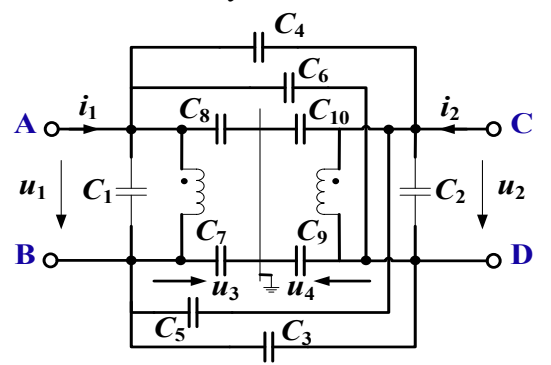

Figure 5. Model of adding new capacitance

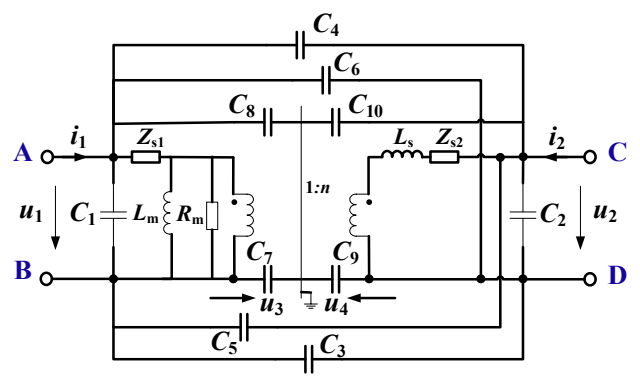

Figure 6. Broadband mechanism model of transformer

Combining the magnetic mechanism model of transformer with its capacitance mechanism model, the broadband mechanism model of transformer is obtained,

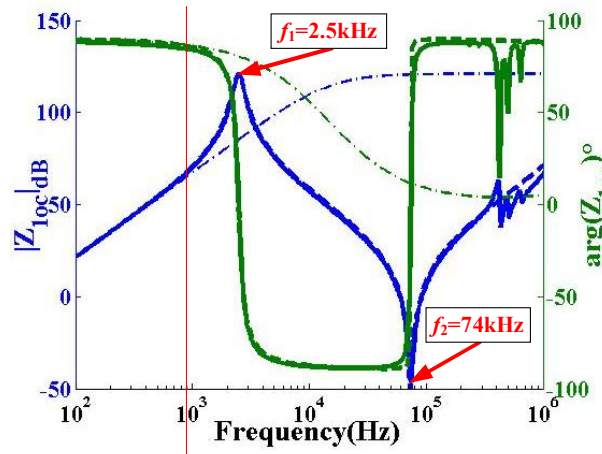

(a)

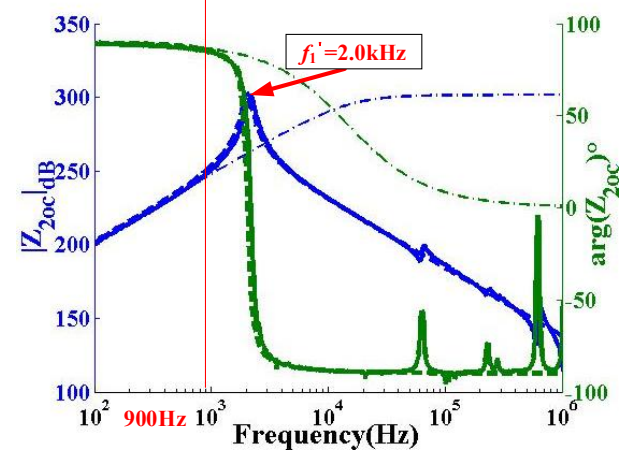

(c) as shown in Figure 6. It is noted that, compared with the magnetic mechanism model shown in Figure 2, the broadband mechanism model shown in Figure 6 replaces the winding resistance for internal impedance, which can reflect the skin effect of the winding at high frequency.

\section{Analysis of model characteristics}

In order to verify the effectiveness of the broadband model of transformer, we use Agilent 4294A impedance analyzer to measure the impedance characteristics of a $500 \mathrm{kV} / 220 \mathrm{kV}$ single-phase transformer under different conditions, such as no-load, short circuit and load (with $125 \mathrm{k}$ ohm resistive load and $40 \mathrm{uH}$ inductive load). At the same time, the circuit simulation of the transformer is also made by using the broadband mechanism model presented previously. Here, the impedance analyzer is connected with the low-voltage winding and the high voltage winding, respectively expressed as "1" and "2"; "OC", "SC", "R" and "L" respectively represent the open circuit, the short circuit, the resistive load and the state of inductive load of the other winding.

The experimental and simulation results are shown in Figure 7 and Figure 8. The thick lines show the measurement results of impedance analyzer. The fine dotted lines represent the simulation results of the magnetic mechanism model of the transformer (the low frequency model of the traditional transformer). The coarse dotted lines represent the simulation results of the broadband mechanism model of the transformer.

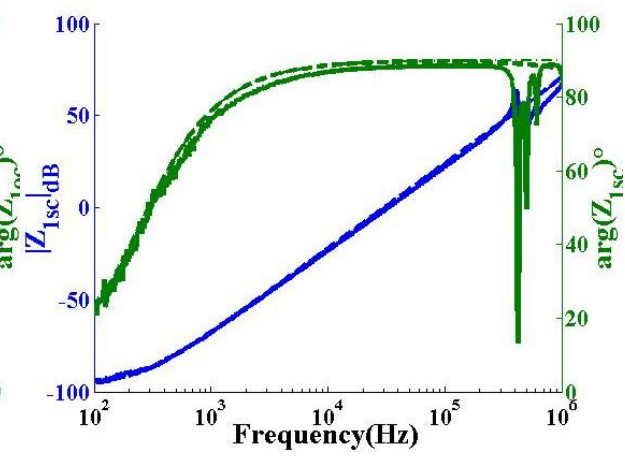

(b)

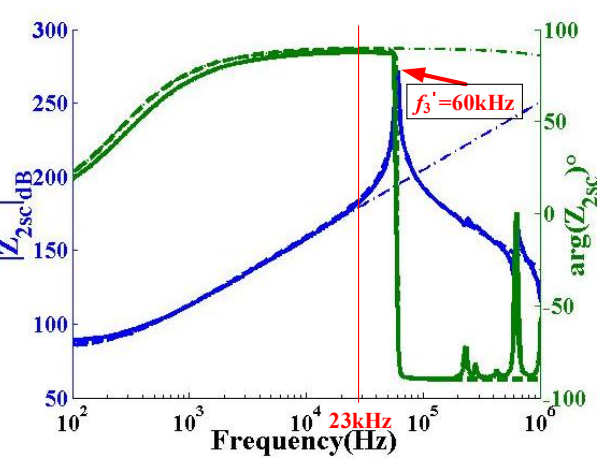

(d)

Figure 7. The amplitude frequency characteristics and phase frequency characteristics of open circuit impedance and short circuit impedance : experimental results (solid line), the simulation results based on the traditional magnetic model (dotted line), the simulation results based on the model of broadband mechanism (thick line).

(a) high voltage winding open circuit (b) high voltage winding short circuit

(c) low voltage winding open circuit (d) low voltage winding short circuit 


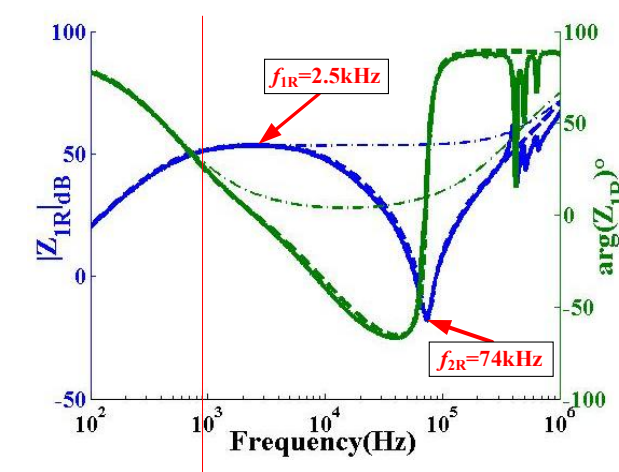

(a)

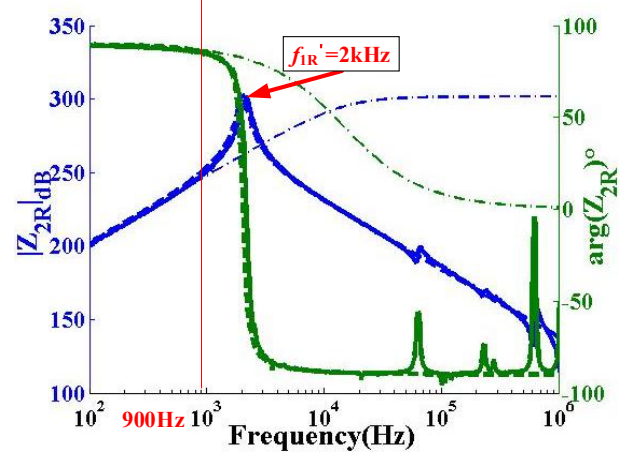

(c)

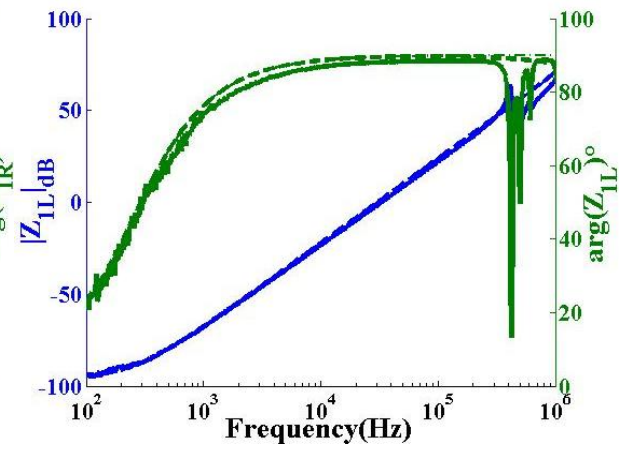

(b)

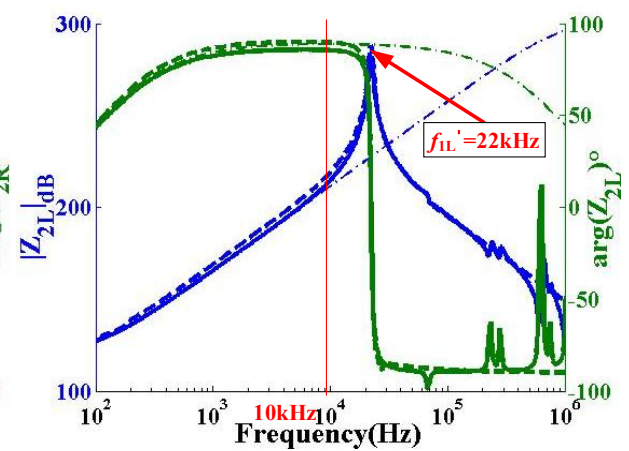

(d)

Figure 8. The amplitude frequency characteristics and phase frequency characteristics of open circuit impedance and short circuit impedance : experimental results (solid line), the simulation results based on the traditional magnetic model (dotted line), the simulation results based on the model of broadband mechanism (thick line).

(a) high voltage winding connected with a resistive load (b) high voltage winding connected with a inductive load

(c) low voltage winding connected with a resistive load (d) low voltage winding connected with a inductive load

From Figure 7 and Figure 8, it can be seen that the simulation results of the traditional transformer model are only consistent with the actual measurement results in the low frequency band, and the simulation results are obviously deviated with the increase of the frequency. The frequency at which the simulation results deviate from the experimental results at $20 \%$ is defined as the critical frequency, as shown in the vertical lines in Figure 7 and figure 8. It can be seen that the critical frequency of the traditional transformer model is about $900 \mathrm{~Hz}$ for the open circuit impedance characteristics of the transformer, which is far lower than the working frequency of the transformer $20 \mathrm{kHz}$. This indicates that the capacitance effect between transformer winding, magnetic core and tank is necessary to be considered. Because some of the capacitors in the model are shorted in the short-circuit state of the transformer, the critical frequency of the Z1sc exceeds $1 \mathrm{MHz}$, and the critical frequency of Z2sc is $23 \mathrm{kHz}$.

\section{Conclusion}

The analysis results show that compared with the traditional transformer model, regardless of the open circuit state or the load state, the simulation results of the transformer model based on wide band mechanism are in good agreement with the actual measurement results, and the critical frequency is as high as $300 \mathrm{kHz}$. The transformer model based on broadband mechanism can accurately reflect the characteristics of magnetic coupling and static coupling of transformer, and also verify the effectiveness of transformer parameter extraction method.

\section{References}

1. W.Xiong, Z.Wenliang, Technical problems of electromagnetic compatibility in power system, High voltage technology, 23,53-58 (1997)

2. V. S. Rashkes, L. D. Ziles, Very high frequency overvoltages at air EHV substations during disconnect switch operations, IEEE on PD, 3,16181623(1996)

3. S.Zhusen, Z.Yufang, Z.Guangzhou, et al. Research on electromagnetic disturbance and protection measures in $500 \mathrm{kV}$ substation (1), high voltage technology,26,16-18(2000)

4. C.Dingxin, Q.Xuedi, Calculation method of magnetic field produced by line current in "wire earth" loop, Proceedings of the Symposium on electromagnetic environment and electromagnetic compatibility of power system, Guangzhou, 2729(2002)

5. W.Xiong, Electromagnetic disturbance and level of $500 \mathrm{kV}$ substation, Symposium on electromagnetic environment and electromagnetic compatibility of power system, Guangzhou,5-8(2002) 